\title{
The role of interference in memory span
}

\author{
CYNTHIA P. MAY \\ University of Arizona, Theson, Arizona \\ LYNN HASHER \\ Duke University, Durham, North Carolina \\ and \\ MICHAEL J. KANE \\ University of South Carolina, Columbia, South Carolina
}

\begin{abstract}
In two experiments, we investigated the possibility that susceptibility to proactive interference (PI) affects performance on memory span measures. We tested both younger and older adults (older adults were tested because of the suggestion that they are differentially susceptible to PI). We used two different span measures and manipulated testing procedures to reduce PI for these tasks. For older adults, span estimates increased with each PI-reducing manipulation; for younger adults, scores increased when multiple PI manipulations were combined or when PI-reducing manipulations were used in paradigms in which within-task PI was especially high. The findings suggest that PI critically influences span performance. We consider the possibility that interference-proneness may influence cognitive behaviors previously thought to be governed by capacity.
\end{abstract}

Several literatures in cognitive psychology suggest that individuals have a limit on the "capacity" available to handle the intellectual workload (e.g., Kahneman, 1973). Central to the issue of limitations have been investigations of the capacity of "working memory" (WM), which is conceptualized as the mental workspace available for the momentary storage and manipulation of activated memory representations (e.g., Baddeley, 1986; Baddeley \& Hitch, 1974; Daneman \& Carpenter, 1980; Just \& Carpenter, 1980, 1992). WM capacity is believed by some to reflect a general cognitive capacity and thus to influence performance on a range of cognitive tasks, including language comprehension, problem solving, and memory (e.g., Engle, Cantor, \& Carullo, 1992; Just \& Carpenter, 1992). Furthermore, WM capacity is thought to vary among individuals and across groups, with the suggestion that these variations in capacity are responsible for individual and group differences in performance on a wide range of intellectual tasks (e.g., Just \& Carpenter, 1980; Kyllonen \& Christal, 1990; Kyllonen \& Stephens, 1990).

Support for the limited capacity view comes largely from studies investigating the relationship between measures of WM capacity and measures of performance on

This research was supported by Grant 4306 from the National Institute on Aging. The authors are particularly grateful to Matt Valenti, who was extremely helpful in the initial stages of this project. The authors also thank Robert Smith, Dyna Michelle, Michael Szarek, Dina Dicenso, Kristi Multhaup, Tamara Rahhal, and Karl Oswald for their assistance with stimulus preparation and data collection. Correspondence should be addressed to C. P. May, Department of Psychology, College of Charleston, 66 George St., Charleston, SC 29424 (e-mail: mayc@cofc.edu). various other cognitive tasks. In these investigations, WM capacity has traditionally been assessed by "complex" memory span tasks, in which participants are presented with information, frequently must manipulate that information, and then recall some or all of it from memory. Span estimates are believed to reflect the maximum amount of information that one can store and process simultaneously, and numerous studies have found span scores to be predictive of performance on other cognitive measures. For example, younger adults with high memory spans are better able to comprehend text (e.g., Daneman \& Carpenter, 1980; Masson \& Miller, 1983), to follow directions (Engle, Carullo, \& Collins, 1991), and to learn new vocabulary (Daneman \& Green, 1986) than are those with low memory spans. As well, older adults typically have lower span estimates relative to younger adults (e.g., Charness, 1987; Gick, Craik, \& Morris, 1988; see Salthouse, 1988, for a review), and many investigators have argued that this reduction in memory capacity is directly responsible for general age-related deficits in cognition (e.g., Craik \& Byrd, 1982; Rabinowitz, Craik, \& Ackerman, 1982; Salthouse, 1988).

We offer an alternative interpretation of the memory span data, one that is grounded in inhibitory models of attention and memory (e.g., Allport, 1989; Hasher \& Zacks, 1988; Navon, 1988a, 1988b). These models posit that a critical component of both attentional and memorial processing is the ability to inhibit irrelevant information so as to enable efficient processing of relevant material. With respect to WM, inhibitory mechanisms serve three important functions: (1) they restrict access into WM to only those items that are relevant, (2) they delete items that were once relevant but are no longer appropriate, and 
(3) they restrain production of strong, or highly probable, responses until they can be evaluated (Hasher, Zacks, \& May, 1999). The deletion function of inhibition may be particularly significant in the consideration of WM and other span tasks, since these tasks often require participants to learn, manipulate, and then recall items on many successive trials. Inhibition of antecedent information is thus critical to retrieving items from only the most recently presented trial. Failure to suppress those items from earlier trials allows them to compete at retrieval with those from the most recent list. Competition among candidate responses is the basic source of proactive interference (PI), which itself is a primary source of momentary forgetting (Underwood, 1957; see Crowder, 1976, for a review). Thus, we posit that span tasks, by their multiple-trial nature, with recall required on each trial only for items in the most recent set, create a tremendous opportunity for the buildup of PI from prior list items. This buildup should be particularly noticeable for people who have a reduced ability to suppress or delete from consideration items from previous lists. The ability to delete no-longer-relevant items reduces the pool of candidates within which competition occurs, and, thus, differences in this ability will contribute to individual differences in span performance. Rather than providing pure measures of a general capacity to store and manipulate or operate on items for a single moment in time, then, memory span measures may well be influenced by individual and group differences in susceptibility to PI.

Consider, for example, the reading span task developed by Daneman and Carpenter (1980). In this task, participants read sentences for comprehension and must remember the final word of each sentence. The sentences are presented in sets, with set size ranging from 2 to 5 sentences. There are usually multiple trials for each set size, and trials are typically presented in ascending order of set size. High span scores are achieved if participants successfully complete trials with high memory loads (i.e., trials for Set Size 4 or 5). Note, though, that because set sizes are presented in ascending order and because PI accumulates over trials, those trials with the greatest memory loads are also those subjected to the greatest PI. Individuals who are unable to suppress or delete items from previous trials will be differentially impaired for large sets, and, thus, differences in span may arise not only from variations in WM capacity but also from differences in the ability to suppress or delete no-longer-relevant information.

Suggestive evidence that interference influences span performance comes from two related lines of work. On the one hand, there are data from work with simple span measures indicating that span scores diminish as the similarity among items increases. For example, span scores in an auditory letter span task are lower when letter sequences are composed of acoustically similar letters (e.g., "FSX") than when letter sequences contain acoustically dissimilar letters (e.g., "JKN"; Conrad \& Hull, 1964). In addition, span scores decrease when list items are drawn from the same category (e.g., digits) relative to when they are drawn from two distinct categories (e.g., digits and words; Young \& Supa, 1941). Finally, in a complex memory span task, span scores diminish when the type of information used in the processing component of the task matches that used in the memory load component (e.g., two verbal tasks-verify a sentence and remember a word), relative to conditions in which different materials are used for the processing versus memory load components (e.g., a spatial task and a verbal task-perform mental rotation and remember a word; Shah \& Miyake, 1996).

In addition to these studies showing an effect of interference on span scores with manipulations of materials, there is also evidence that individual differences in interference-proneness may affect performance on span tasks. Many early findings were suggestive of the possibility that PI susceptibility may be a source of span differences (e.g., Jensen, 1964; Rosner, 1972; see Dempster, 1981, for a review), and a set of studies by Dempster and Cooney (1982) examined the relation between span performance and interference-proneness using a forward digit span task and two separate measures of PI. Although the correlations between the span and interference measures failed to reach significance (a finding that may reflect relatively low power, as well as floor/ceiling effects), there was evidence that low-span participants showed reliably greater PI than high-span participants.

In the present experiments, we sought direct evidence that PI is a significant determinant of span performance using two span tasks: the Daneman and Carpenter (1980) reading span task (Experiments 1 and 2 ) and the backward digit span task (Experiment 2). In two experiments, we manipulated the interference-inducing qualities of the memory span task itself: First, we varied the presentation format, with half of the participants performing the span task in the standard, ascending format (beginning with Set Size 2 and progressing to larger sets), and the other half performing the span task in a descending format (beginning with the largest sets and progressing to smaller sets). Second, we created contextual shifts after each span trial using unique filler tasks in an attempt to make each trial distinctive and so reduce PI across trials. We note that while these manipulations were intended to attenuate PI across trials in the span task, in no way did we expect them to eliminate PI entirely, since there are numerous potential sources for PI (e.g., within-trial interference from sentence endings or from the sentences themselves) in addition to the PI that builds across trials. Our aim was to demonstrate that even modest reductions in PI can affect span performance.

As a further way of assessing the role of PI in span tasks, we tested older adults along with college students because of strong evidence indicating that older adults are differentially susceptible to PI relative to younger adults (e.g., Traxler, 1973; Winocur \& Moscovitch, 1983; see also Kane \& Hasher, 1995, for a review). We expected, then, that our two PI manipulations would have 
a particularly large impact on the span performance of older adults. As well, we explored the possibility that agerelated deficits in span performance reflect, at least in part, age differences in interference-proneness, which may in turn be due to the inability to effectively suppress no-longer-relevant information (e.g., Hasher \& Zacks, 1988; McDowd, Oseas-Kreger, \& Filion, 1995). If agerelated span deficits result from the competition among relevant and irrelevant items in WM, as well as (or rather than) from a diminished WM capacity, then age differences in span performance should be attenuated with manipulations that reduce PI.

By contrast, from a pure capacity interpretation of span measures, our PI-reducing manipulations should have little impact on span performance. That is, the amount of information an individual can store and process simultaneously should remain relatively stable despite changes in the format of presentation or with the addition of distinct breaks after sets. To preview our findings, the data indicate that older adults benefit from all of the reductions in $\mathrm{PI}$ and that younger adults benefit when PI is very high or when multiple methods of PI reduction are used. In short, the results suggest that interference susceptibility is an important determinant of span-task performance and the age differences therein.

\section{EXPERIMENT 1}

In Experiment 1, we explored whether PI affects performance in the Daneman and Carpenter (1980) reading span task. We tested younger and older adults in two different formats of the reading span task: a standard one and one that, from an inhibitory perspective, reduces PI. In the standard format, we tested participants with the traditional ascending format of the reading span task. In an alternative, "descending" format, we tested participants with the very same materials but began with the largest sets and progressed to smaller sets. Our expectation was that participants who were tested in the descending format and thus received the large sets earlier and so with minimal PI would demonstrate higher spans than those tested in the standard format who received the large sets under conditions of greater PI, since the larger sets followed many short sets. Furthermore, we predicted that reductions in PI would also lead to reductions in age differences in span.

\footnotetext{
Method

Participants. Sixty-five younger adults (18-21 years of age) and 40 older adults $(60-75$ years of age) participated in this experiment. Younger adults were undergraduates at the University of Arizona, and older adults were community-dwelling volunteers in Tucson, Arizona.

Materials. Forty-five sentences were randomly selected from the set of 88 developed by Daneman and Carpenter (1980), and those sentences were modified so that each one was 10-13 words in length. The sentences were then randomly assigned to create five lists of two sentences each, five lists of three sentences each, and five lists of four sentences each. Two different formats were con-
}

structed: (1) the standard format, which began with five lists of Set Size 2 and then progressed to five lists of Set Size 3 and then to Set Size 4; and (2) the descending format, which began with five lists of Set Size 4, then Set Size 3, then Set Size 2. Within each set size, presentation of a given set of sentences in the first, second, third, fourth, or fifth list was counterbalanced across participants in each age group and presentation format. Two familiar sentences were presented as practice items (i.e., "Mary had a little lamb" and "Jack and Jill went up the hill") so they could be easily distinguished from the test sentences and would thus produce minimal interference.

Procedure. Instructions for the sentence span task closely followed those described by Daneman and Carpenter (1980). For each trial, the participants read aloud sentences typed individually on index cards, and they tried to understand each sentence and then to remember the last word of each sentence. As soon as the participants finished reading each sentence, the next sentence was presented immediately to limit rehearsal of the final words. At the end of each trial, a white card cued the participants to recall all sentenceending words from that trial.

After reviewing the instructions, the experimenter read the two practice sentences aloud and demonstrated the appropriate recall for the practice trial. Thirty-five younger adults and 20 older adults performed the span task in the standard format, and 30 younger and 20 older participants performed the span task in the descending format. Every participant received all 15 trials in the experiment, regardless of performance. After finishing the span task, the participants completed Version 3 of the Extended Range Vocabulary Test (ERVT; Educational Testing Service, 1976).

\section{Results}

For all analyses reported in Experiments 1 and 2, the alpha level was .05 .

Participants. Younger adults $(M$ age $=19.0$ years, range $=18-24$ years) averaged 12.0 years of education and a score of 13.0 on the ERVT. Older adults $(M$ age $=$ 67.4 years, range $=62-75$ years $)$ had significantly more years of education $(M=14.3$ years) $[F(1,101)=17.4$, $\left.M S_{\mathrm{e}}=6.2\right]$ and scored significantly higher on the ERVT $(M=30.2)$ than younger adults $\left[F(1,101)=135.1, M S_{\mathrm{e}}=\right.$ 54.5]. There were no main effects of span format and no interactions of span format $\times$ age on any of these demographic variables $(F \mathrm{~s}<1)$.

Scoring. The span data were scored in two different ways. First, we used the traditional scoring procedure developed by Daneman and Carpenter (1980), in which a participant's span was equivalent to the highest set size at which they correctly recalled all of the final words for three out of the five trials. The participants received partial credit (of .5) if they recalled all of the final words for two of the five trials at that set size. Scoring for all participants, regardless of presentation format, began with Set Size 2, and each participant had to reach the criteria for Set Size 2 before performance on Set Size 3 was considered, and so on. The second measure we used was an items score, calculated as the total number of words recalled when only fully correct trials were considered (e.g., Engle et al., 1992).

Data analyses. Means and standard deviations for the traditional span score and the items score are presented in Table 1. For each dependent measure, a $2 \times 2$ analysis of variance (ANOVA) was conducted to determine the 
Table 1

Means and Standard Deviations for

Reading Span Scores in Experiments 1 and 2

\begin{tabular}{|c|c|c|c|c|c|c|c|c|}
\hline \multirow[b]{3}{*}{ Format } & \multicolumn{4}{|c|}{ Experiment 1} & \multicolumn{4}{|c|}{ Experiment 2} \\
\hline & \multicolumn{2}{|c|}{ Standard Score } & \multicolumn{2}{|c|}{ Items Score } & \multicolumn{2}{|c|}{ Standard Score } & \multicolumn{2}{|c|}{ Items Score } \\
\hline & $M$ & $S D$ & $M$ & $S D$ & $M$ & $S D$ & $M$ & $S D$ \\
\hline \multicolumn{9}{|c|}{ Younger Adults } \\
\hline Standard & 3.1 & 0.5 & 23.3 & 8.1 & 3.2 & 0.6 & 24.1 & 7.8 \\
\hline Descending & 2.9 & 0.5 & 21.4 & 6.3 & 3.2 & 0.6 & 26.8 & 8.2 \\
\hline \multicolumn{9}{|c|}{ Older Adults } \\
\hline Standard & 2.6 & 0.7 & 17.1 & 8.1 & 3.0 & 0.7 & 24.7 & 10.8 \\
\hline Descending & 3.0 & 0.4 & 23.3 & 7.0 & 2.8 & 0.6 & 22.2 & 9.2 \\
\hline
\end{tabular}

effects of age and span format on performance, and the results indicate identical patterns for the two scores. ${ }^{1}$ There were neither main effects of age nor main effects of span format $(p s>.15)$, but there was a significant age $\times$ span format interaction $\left[F(1,101)=7.4, M S_{\mathrm{e}}=55.0\right]$. Older adults performed reliably better in the descending format than in the standard format $[F(1,38)=7.0]$, but this difference across formats was not significant for younger adults $[F(1,63)=1.1, p>.3]$. As a result, there was a reliable age difference for the participants tested with the standard format $[F(1,53)=9.1]$, a finding that replicates a substantial number of studies on cognitive gerontology; however, this age difference was not reliable for either score in the descending format $(F \mathrm{~s}<1.5)$.

\section{Discussion}

Memory span tasks typically begin with tests of the smallest set sizes first, followed by tests of successively larger set sizes. For any participants who have difficulty cutting off the past (or suppressing no-longer-relevant information), retrieval difficulties should be greatest as the number of successive test trials increases, which coincides with larger set sizes in the span task. Other research has suggested that older adults are indeed less able to stop processing the recent past than are younger adults (e.g., Hamm \& Hasher, 1992; Hartman \& Hasher, 1991; Zacks, Radvansky, \& Hasher, 1996), whether they are explicitly or only tacitly instructed to do so (Zacks et al., 1996). Because older adults do not successfully cut off the past, they create a retrieval pool of candidate items on any one trial that will include both currently relevant items and no-longer-relevant items, creating for themselves a larger set size or "fan" (Anderson, 1983; Watkins \& Watkins, 1975) than is the case for others with more efficient suppression mechanisms. These larger fans then disrupt retrieval (see also Gerard, Zacks, Hasher, \& Radvansky, 1991), an effect most plainly seen in span tasks when the set sizes get larger, at least for participants unable to successfully suppress information that is no longer relevant.

In Experiment 1, we set up a procedure designed to minimize the impact of prior (smaller) sets on the recall of the most recent (largest) sets by presenting the larger sets first to half the participants. As might be expected from a literature showing superior suppression effects for young adults (e.g., Hasher et al., 1999), this manipulation made little difference in their span performance. For older adults, however, there was a substantial improvement in performance; in fact, the descending span score of older adults did not differ from the span scores of young adults. We note that the change in performance for older adults cannot be attributed to changes in capacity or activation, since it is difficult to see how list structure could impact on that presumed mechanism. What these data clearly suggest, however, is that traditional memory span tasks do indeed permit the operation of PI, and this will reduce the apparent span measure for those, like older adults, who have difficulty cutting off the past.

\section{EXPERIMENT 2}

In Experiment 2, we used two tasks to further investigate the impact of PI on span performance. The first task replicated the ascending/descending procedure of Experiment 1 , but this time on a different span task, the backward digit span from the WAIS-R. This task was chosen because of its widespread use and because it uses a very small set of items (digits 1-9) repeatedly in different combinations, thus maximizing similarity across sets and so (presumably) maximizing PI (e.g., Kausler, 1974). Given the very high level of potential PI here, we anticipated that both older and younger adults might benefit from the descending span manipulation.

In the second task, we returned to the reading span test, but now we combined the ascending/descending procedure with an additional procedure known to reduce PI-that is, inducing a contextual change after each test trial (e.g., Wickens \& Cammarata, 1986; Wickens \& Gittis, 1974). For the second task, we inserted unique filler tasks after each trial in the Daneman and Carpenter (1980) reading span task, and we tested half of all participants in the ascending format and half in the descending format. Note that, in Experiment 2, we sampled from the same groups of younger and older adults as in Experiment 1, and, aside from the new break manipulation, all other materials and procedures were identical to those in Experiment 1 . By comparing the newly collected data with those reported in Experiment 1, we were able to investigate two related questions: First, what is the effect of in- 
Table 2

Means and Standard Deviations for Backward Digit Span Scores in Experiment 2

\begin{tabular}{llclll}
\hline & \multicolumn{2}{c}{ Standard Score } & \multicolumn{2}{c}{ Items Score } \\
\cline { 2 - 5 } Format & $M$ & $S D$ & & $M$ & $S D$ \\
\hline \multirow{2}{*}{ Younger Adults } & & \\
Standard & 15.0 & 3.4 & 26.7 & 8.9 \\
Descending & 16.3 & 3.0 & 29.9 & 7.8 \\
Standard & \multicolumn{2}{c}{ Older Adults } & & \\
Descending & 13.5 & 3.9 & 23.1 & 9.7 \\
\hline
\end{tabular}

ducing a contextual change after each trial on span performance, and does this effect differ across age groups? If the span measure is indeed influenced by PI, the addition of the breaks should act to reduce PI relative to conditions in which no breaks are included. This should particularly be the case when no other PI-reducing manipulations are engaged (e.g., ascending format), as well as for those who have difficulty inhibiting the recent past (in this study, older adults). Thus, for the ascending format of the reading span task, we expected that the addition of contextual breaks would enhance the performance of older adults relative to the no-break condition used in Experiment 1 .

Because younger adults are relatively successful in suppressing the past, we expected to replicate the finding from Experiment 1 that a single manipulation intended to reduce PI does not affect span performance for younger adults; thus, we expected the performance of younger adults tested in the ascending break condition to be similar to that of the younger adults tested in the ascending no-break condition used in Experiment 1.

The second question addressed here concerns the impact of combining PI-reducing manipulations on span performance for younger and older adults. Data from Experiment 1 suggest that older adults, but not younger adults, benefit from a single reduction in PI; here, we explored whether older adults show an additional benefit when multiple PI manipulations were employed, and whether younger adults might also show a reliable benefit when PI reductions were combined.

\section{Method}

Participants. Sixty new younger adults and 50 new older adults were selected from the same populations described in Experiment 1 .

Materials. Materials for the digit span test were adapted from the backward digit span test of the WAIS-R. In this test, the participants heard a string of digits and then repeated them in reverse order. The materials we used were identical to those in the backward digit span test, with the exception that our maximum set size was $6 .^{2}$

The sentences for the reading span task (and their groupings into sets of 2,3, and 4 sentences) were identical to those in Experiment 1 . In addition, we selected 14 unique nonverbal filler tasks from the Kit of Factor-Referenced Tests (Educational Testing Service, 1976) to be completed after each of the span trials. These tasks included perceptual matching, pattern recognition, and symbol generation and were demanding and engaging.

Procedure. The participants began with the backward digit span task. Half of the participants in each age group began with strings of two digits and proceeded to strings of six digits (standard format); the remaining participants began with strings of six and proceeded to strings of two (descending format). Regardless of format, each participant received two consecutive strings at each set size, for a total of 20 strings.

After completing the backward digit span task, the participants performed the version of the reading span task that included unique tasks between trials (called the "break" version). The procedures were identical to those described in Experiment 1, with the addition of the filler tasks after each trial. Half of the participants in each age group were tested with the standard format, and half were tested with the descending format. The participants first received reading span instructions and then the instructions for the break tasks. The participants again read sets of sentences and reported the final words of those sentences. After the recall for each trial, the participants received one of the break tasks for $90 \mathrm{sec}$ before proceeding to the next trial.

\section{Results and Discussion}

Participants. Younger adults $(M$ age $=20.3$ years, range $=18-24$ years) averaged 13.2 years of education and a score of 16.0 on the ERVT. Older adults $(M$ age $=$ 69.6 years, range $=60-75$ years $)$ had significantly more years of education $(M=14.9$ years $)[F(1,106)=19.6$, $\left.M S_{\mathrm{e}}=4.0\right]$ and scored significantly higher on the ERVT $(M=32.1)$ than younger adults $\left[F(1,106)=111.1, M S_{\mathrm{e}}=\right.$ $64.2]$. There were no main effects of span format or digit format, nor were there any reliable interactions on the demographic variables.

Backward digit span. The digit span data were scored in two ways. First, we used the standard method of scoring, in which the span score was equivalent to two times the number of strings that the participants correctly recalled in reverse order. Second, we calculated an item digit score, which was equivalent to the total number of digits that were correctly recalled when only items from correct trials were counted. Means for both scores are displayed in Table 2.

Separate 2 (digit format) $\times 2$ (age) ANOVAs indicated similar findings across the scores, and so here we report analyses for the items score. There was a main effect of digit format $\left[F(1,106)=5.6, M S_{\mathrm{e}}=74.9\right]$, with higher scores for the participants tested in the descending format than for those tested in the standard format. There were marginal age effects $[F(1,106)=3.0, p=.09]$, with younger adults recalling more digits than older adults. The digit format $\times$ age interaction was not significant $(F<1)$, indicating that both age groups benefited from the descending format relative to the standard format. The benefit for younger adults here but not in Experiment 1 for reading span may be the result of heightened overall interference caused by the high interlist similarity (and so competition) among digits.

Reading break span. The measures used to assess performance on the reading break span task were identical to those employed in Experiment 1 (see Table 1). Our 


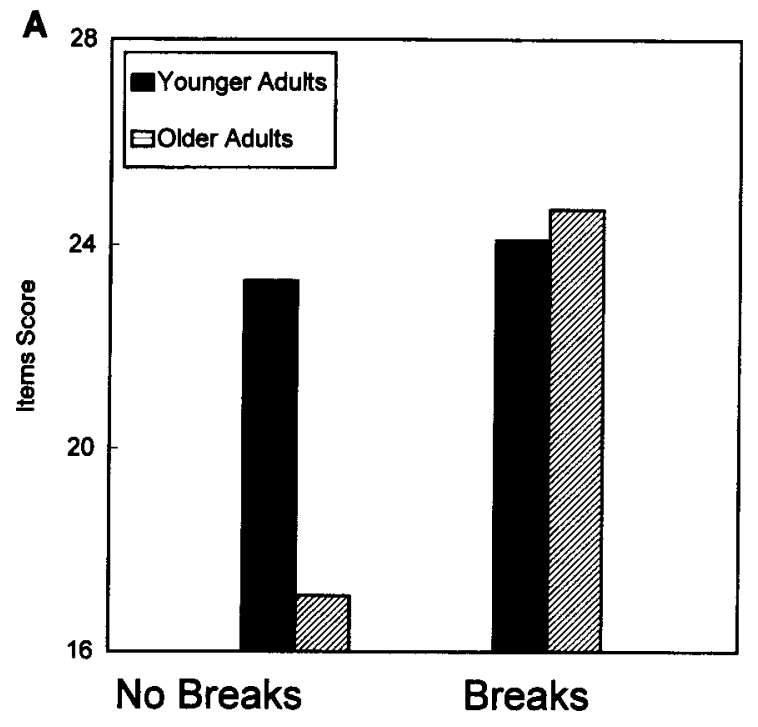

Ascending Format
B

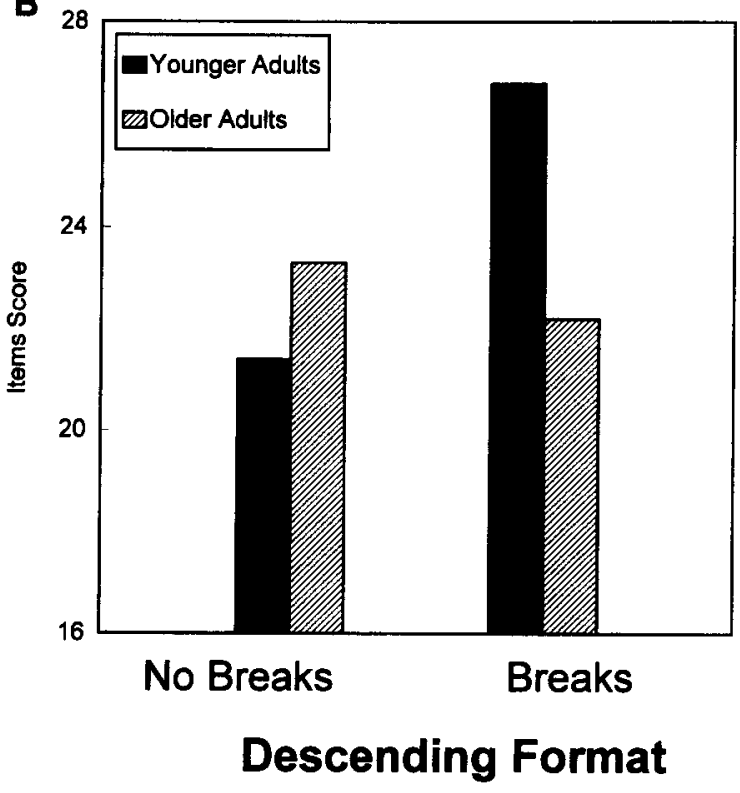

Figure 1. (A) Items score for younger and older adults tested in the ascending format both without breaks (Experiment 1) and with distinctive breaks between trials (Experiment 2). (B) Items score for younger and older adults tested in the descending format both without breaks (Experiment 1) and with distinctive breaks between trials (Experiment 2).

analyses were guided by two theoretical questions outlined earlier: (1) Does the addition of unique breaks enhance span performance relative to the traditional span task, and is the effect similar for younger and older adults? and (2) Is the effect of combined PI reductions greater than that of a single PI reduction for either age group? Because these questions involved both within- and across-experiment comparisons, the data from Experiment 2 were combined with those from Experiment 1 and submitted to a 2 (age) $\times 2$ (span format) $\times 2$ (experiment) ANOVA. ${ }^{3}$

For the items score, there were main effects of age $\left[F(1,207)=3.3, M S_{\mathrm{e}}=0.67 .8\right]$ and of experiment $[F(1,207)=7.7]$, with younger adults generally showing higher scores than older adults, and with greater span scores for the participants tested in the break span relative to those tested in the traditional reading span. No other main effects or two-way interactions were significant, but the age $\times$ experiment $\times$ span format interaction was reliable $[F(1,207)=8.5]$.

As can be seen in Figure 1, this three-way interaction may be best understood as reflecting differences in the susceptibility to PI across age groups and, consequently, in the benefit obtained from the two PI manipulations used across the experiments. To preview, the findings suggest that older adults are quite susceptible to PI, showing relatively poor performance when PI is maximal (i.e., ascending format without breaks), and they demonstrate significant benefits from even small reductions in PI (i.e., the addition of distinctive breaks or the descending format). Younger adults, by contrast, are relatively more resistant to PI than are older adults, with their perfor- mance changing only when multiple PI reductions are combined (i.e., descending format with breaks).

Consider first Figure 1A, which shows the scores for the traditional ascending format both without breaks (Experiment 1) and with breaks (Experiment 2). In the traditional span task without breaks, older adults are reliably impaired relative to age mates tested in all other conditions $\left[F(1,88)=7.7, M S_{\mathrm{e}}=67.8\right]$ and relative to younger adults in general $[F(1,143)=11.8]$. Older adults' performance improves significantly, though, with any reduction in PI: Scores are reliably higher when older adults are tested with the addition of breaks alone even in the ascending format $[F(1,43)=9.5]$ or (now see Figure 1B) with use of the descending format alone $[F(1,38)=6.2]{ }^{4}$ Moreover, when either of these single PI manipulations is employed, the performance of older adults does not differ from that of younger adults in the respective singlemanipulation conditions $(F \mathrm{~s}<1)$. Thus, with modest reductions in PI, older adults perform at the levels of younger adults. However, there is a limit to the extent of older adults' improvement; combining the break procedure with the descending procedure (i.e., descending format, Experiment 2) has no additional benefit for older adults over and above the benefits derived from either manipulation alone $(F \mathrm{~s}<1)$.

Consistent with the notion that younger adults are relatively resistant to PI, span performance does not differ for younger adults across the traditional span (i.e., ascending without breaks) and single PI manipulation conditions (i.e., ascending with breaks and descending without breaks) $(F \mathrm{~s}<1.5)$. For the sort of materials used in reading span tasks, younger adults benefit only when PI 
manipulations are combined: With both the descending and break procedures, younger adults show a reliable improvement relative to with the single-manipulation conditions $[F(1,88)=4.8]$.

\section{GENERAL DISCUSSION}

Three general conclusions emerge from the reading span tasks used in Experiments 1 and 2. First, as suggested in Experiment 1, PI can influence performance on the reading span task; PI-reducing procedures did act to improve span scores in many instances. Second, the impact of PI is greater for older adults than for younger adults. Older adults showed relatively poor span performance when PI was maximal (standard, ascending format without breaks), and they benefited from two separate PIreducing manipulations, elevating their performance to the level of younger adults. By contrast, younger adults improved only when PI reductions were combined, suggesting that they are relatively resistant to PI and so can only benefit from experimental manipulations that reduce PI greatly. Third, the present data suggest that older adults' differential susceptibility to PI cannot account entirely for age differences in span performance. In the condition that arguably involved the least PI (i.e., descending format with breaks), age differences in span were reliable, suggesting that although differences in PI may contribute to age differences in span estimates, other sources are also responsible. The obvious candidate for at least one source of the age differences is, of course, general capacity.

The reading span data join with the digit span data to suggest that operations that reduce interference can boost estimates of WM span. Taken together, the data from Experiments 1 and 2 suggest that younger adults and older adults can benefit from PI manipulations, specifically in contexts with high levels of materials-induced PI (e.g., digit span task) or when PI reductions are particularly robust (e.g., descending format with breaks).

We turn now to the implications of the present findings. Are we limited in our capacity to store and process information at any given moment? Perhaps so. However, the widely held assumption that WM span tasks are a reasonably good measure of that capacity is challenged by the present data, which suggest that WM span performance is at least partially determined by the presence of interference in the task. In three separate tasks, we varied testing procedures in an effort to reduce PI in standard WM span tasks. The data indicate that span performance improved for older adults with each manipulation intended to reduce Pl (i.e., descending format and distinctive breaks between tasks), and span performance improved for younger adults when PI reductions were combined (descending format and distinctive breaks) or when the tobe-remembered material was highly similar across trials (digit span).

By a capacity view, WM span estimates should have remained constant across our manipulations, given that the materials, memory loads, and processing demands were consistent across high- and low-PI conditions. The finding of higher span scores for low-PI conditions (relative to those for high-PI conditions) thus supports the suggestion that performance on WM span tasks is determined at least in part by susceptibility to interference and that span tasks do not provide pure indices of processing capacity (see also Lustig \& Hasher, 1998). ${ }^{5}$

The fact that PI contributes to span performance raises a number of interesting possibilities with respect to previously held assumptions based on memory span performance. For example, the present data suggest an alternative to the popular belief that age-related deficits in cognitive functioning are due primarily to reductions in WM capacity. The evidence for deficits in WM capacity with age stems primarily from findings of smaller memory spans for older adults relative to younger adults (e.g., Salthouse, 1988), and, clearly, these span differences are exacerbated by older adults' differential susceptibility to PI. Because age differences in span are at least in part a result of age-related deficits in suppressing no-longerrelevant information, evidence for a relation between span performance and other cognitive measures with age may indicate that the ability to cut off the past, rather than general mental capacity, is an important determinant of age differences in many cognitive behaviors. This possibility is consistent with an inhibitory-deficit view of aging (e.g., Hasher \& Zacks, 1988), which suggests that age-related impairments in the suppression of no-longer-relevant information can have global consequences for cognitive functioning.

In addition, the results here may have implications for a large literature suggesting that WM capacity reflects a general cognitive capability that determines performance on a range of cognitive tasks. Support for this assumption has come from studies showing correlations between span measures and various cognitive processes, such as reasoning, comprehension, and problem solving (e.g., Daneman \& Carpenter, 1980; Daneman \& Merikle, 1996; Fry \& Hale, 1996; Shute, 1991). The present data, which show that WM span tasks may measure interference-proneness in addition to capacity for both older and younger adults, suggest that resistance to interference may also affect performance on many cognitive tasks. Indeed, other studies show that individual differences in susceptibility to PI are predictive of scores on standard achievement tests (Borkowski, 1965; Dempster, 1985); our findings raise the possibility that susceptibility to PI may be related to performance on a wide variety of mental tasks.

\section{REFERENCES}

Allport, A. (1989). Visual attention. In M. I. Posner (Ed.), Foundations of cognitive science (pp. 631-682). Cambridge, MA: MIT Press. ANDERSON, J. R. (1983). The architecture of cognition. Cambridge, MA: Harvard University Press.

BaDdeley, A. (1986). Working memory. Oxford: Oxford University Press.

Baddeley. A. D., \& Hitch, G. (1974). Working memory. In G. H. Bower (Ed.), The psychology of learning and motivation: Advances 
in research and theory (Vol. 8, pp. 47-89). New York: Academic Press.

BORKOWSKI, J. G. (1965). Interference effects in short-term memory as a function of level of intelligence. American Journal of Mental Deficiency, 70, 458-465.

Charness, N. (1987). Component processes in bridge bidding and novel problem-solving tasks. Canadian Journal of Psychology, 41, 223-243.

ConRad, R., \& Hull, A. J. (1964). Information, acoustic confusion and memory span. British Journal of Psychology, 55, 429-432.

ConWAY, A., \& ENGLE, R. (1994). Working memory and retrieval: A resource-dependent inhibition model. Journal of Experimental Psychology: General, 123, 354-373.

Craik, F. I. M., \& BYRD, M. (1982). Aging and cognitive deficits: The role of attentional processes. In F. I. M. Craik \& S. Trehub (Eds.), Aging and cognitive processes (pp. 191-211). New York: Plenum.

CROWDER, R. G. (1976). Principles of learning and memory. Hillsdale, NJ: Erlbaum.

Daneman, M., \& Carpenter, P. A. (1980). Individual differences in working memory and reading. Journal of Verbal Learning \& Verbal Behavior, 19, 450-466.

Daneman, M., \& GREen, I. (1986). Individual differences in comprehending and producing words in context. Journal of Memory \& Language, $25,1-18$.

Daneman, M., \& Merikle, P. M. (1996). Working memory and language comprehension: A meta-analysis. Psychonomic Bulletin $\&$ Review, 3, 422-433.

DEMPSTER, F. N. (1981). Memory span and short-term memory capacity: A developmental study. Journal of Experimental Child Psychology, 4, 419-431.

DEMPSTER, F. N. (1985). Proactive interference in sentence recall: Topicsimilarity effects and individual differences. Memory \& Cognition, 13, 81-89.

DEMPSTER, F. N., \& COONEY, J. B. (1982). Individual differences in digit span, susceptibility to proactive interference, and aptitude/achievement test scores. Intelligence, 6, 399-416.

Educational Testing Service (1976). Kit of factor-referenced tests. Princeton, NJ: Author

Engle, R. W., Cantor, J., \& Carullo, J. J. (1992). Individual differences in working memory and comprehension: A test of four hy potheses. Journal of Experimental Psychology: Learning, Memory, \& Cognition, 18, 972-992.

Engle, R. W., Carullo, J. J., \& Collins, K. W. (1991). Individual differences in working memory for comprehension and following directions. Journal of Educational Research, 84, 253-262.

ENGLe, R. W., Conway, R., Tuholski, S. W., \& Shisler, R. J. (1995). A resource account of inhibition. Psychological Science, 6, 122-125.

FRY, A., \& HALE, S. (1996). Processing speed, working memory, and fluid intelligence: Evidence for a developmental cascade. Psychological Science, 7, 237-241.

Gerard, L., Zacks, R. T., Hasher, L., \& Radvansky, G. A. (1991). Age deficits in retrieval: The fan effect. Journal of Gerontology: Psychological Sciences, 46, 131-136.

Gick, M. L., CraIK, F. I. M., \& Morris, R. G. (1988). Task complexity and age differences in working memory. Memory \& Cognition, 16 , 353-361.

HAMm, V. P., \& HaSher, L. (1992). Age and the availability of inferences. Psychology \& Aging, 7, 56-64.

Hartman, M., \& Hasher, L. (1991). Aging and suppression: Memory for previously relevant information. Psychology \& Aging, 6, 587-594

HASHER, L., \& ZACKS, R. T. (1988). Working memory, comprehension, and aging: A review and a new view. In G. H. Bower (Ed.), The psychology of learning and motivation (Vol. 22, pp. 193-225). San Diego: Academic Press.

HAShER, L., ZACKS, R. T., \& MAY, C. P. (1999). Inhibitory control, circadian arousal, and age. In A. Koriat \& D. Gopher (Eds.), Attention and performance XVII (pp. 653-675). Cambridge, MA: MIT Press.

JENSEN, A. R. (1964). Individual differences in learning: Interference factor (Final Rep., Cooperative Research Project No. 1897). Washington, DC: U.S. Department of Health, Education, and Welfare, Office of Education.

Just, M. A., \& CARPENTER, P. A. (1980). A theory of reading: From eye fixations to comprehension. Psychological Review, 87, 329-354.

Just, M. A., \& CARPENTER, P. A. (1992). A capacity theory of comprehension: Individual differences in working memory. Psychological Review, 99, 122-149.

Kahneman, D. (1973). Attention and effort. Englewood Cliffs, NJ: Prentice-Hall.

KANE, M. J., \& HAShER, L. (1995). Interference. In G. Maddox (Ed.), Encyclopedia of aging (2nd ed., pp. 514-516). New York: SpringerVerlag.

KAUSLER, D. H. (1974). Psychology of verbal learning and memory. New York: Academic Press.

Kyllonen, P. C., \& Christal, R. E. (1990). Reasoning ability is (little more than) working-memory capacity?! Intelligence, 14, 389-433.

Kyllonen, P. C., \& STEPhENS, D. L. (1990). Cognitive abilities as determinants of success in acquiring logic skill. Learning \& Individual Differences, 2, 129-160.

LustiG, C., \& HASHER, L. (1998), Working memory span: The role of interference from the past. Manuscript submitted for publication.

MASSON, M. E. J., \& MilLer, J. A. (1983). Working memory and individual differences in comprehension and memory of text. Journal of Educational Psychology, 75, 314-418.

McDowd, J. M., Oseas-Kreger, D. M., \& Filion, D. L. (1995). Inhibitory processes in cognition and aging. In F. N. Dempster \& C. J. Brainerd (Eds.), Interference and inhibition in cognition (pp. 363401). San Diego: Academic Press.

NAvon, D. (1988a). The importance of being visible: On the role of attention in a mind viewed as an anarchic intelligence system. I. Basic tenets. European Journal of Cognitive Psychology, 1, 191-213.

NAVON, D. (1988b). The importance of being visible: On the role of attention in a mind viewed as an anarchic intelligence system. II. Basic tenets. European Journal of Cognitive Psychology, 1, 215-238.

Rabinowitz, J. C., Craik, F. I. M., \& Ackerman, B. P. (1982). A processing resource account of age differences in recall. Canadian Journal of Psychology, 36, 325-344.

ROSNER, S. R. (1972). Primacy in preschooler's short-term memory: The effects of repeated tests and shift-trials. Journal of Experimental Child Psychology, 13, 220-230.

SALthouse, T. A. (1988). The role of processing resources in cognitive aging. In M. L. Howe \& C. J. Brainerd (Eds.), Cognitive development in adulthood (pp. 185-239). New York: Springer-Verlag.

ShaH, P., \& MiYAKE, A. (1996). The separability of working memory resources for spatial thinking and language processing: An individual differences approach. Journal of Experimental Psychology: General, $125,4-27$

SHUTE, V. J. (1991). Who is likely to acquire programming skills? Journal of Educational Computing Research, 7, 1-24.

Traxler, A. J. (1973). Retroactive and proactive inhibition in young and elderly adults using an unpaced modified recall test. Psychological Reports, 32, 215-222.

UNDERWOOD, B. J. (1957). Interference and forgetting. Psychological Review, 64, 49-60.

WATKINS, O. C., \& WATKINS, M. J. (1975). Build-up of proactive inhibition as a cue-overload effect. Journal of Experimental Psychology: Human Learning \& Memory, 1, 442-452.

Wickens, D. D., \& Cammarata, S. A. (1986). Response class interference in STM. Bulletin of the Psychonomic Society, 24, 266-268.

WiCKENS, D. D., \& GiTTIS, M. M. (1974). The temporal course of recovery from interference and degree of learning in the Brown-Peterson paradigm. Journal of Experimental Psychology, 102, 1021-1026.

Winocur, G., \& Moscovitch, M. (1983). Paired associate learning in institutionalized and noninstitutionalized old people: An analysis of interference and context effects. Journal of Geronotology, 38, 455464.

YounG, C. W., \& SUPA, M. (1941). Mnemic inhibition as a factor in the 
limitation of the memory span. American Journal of Psychology, 54, 546-552.

Zacks, R. T., Radvansky, G. A., \& Hasher, L. (1996). Studies of directed forgetting in older adults. Journal of Experimental Psychology: Learning, Memory, \& Cognition, 22, 143-156.

\section{NOTES}

1. In Experiments 1 and 2, the pattern of findings was similar for the traditional span score and the items score; thus, we report only the analyses for the items score.

2. Pilot testing indicated that very few participants of either age group could accurately report more than six digits in reverse format; thus, six was chosen as the maximum digit set size.

3. This combination was done after ensuring that there were no withinage-group differences in ERVT or age across Experiments 1 and 2.

4. Note that the improvement demonstrated by older adults in the ascending break task relative to the ascending standard task suggests that the benefit observed in span performance is not simply a "fatigue ef- fect," for the following reason: The duration of the break span task was longer than that of the standard task, and the break task required the participants to alternate between the memory span task and a number of different, demanding visual discrimination tasks. Because the addition of these tasks would have increased, rather than decreased, fatigue effects, the finding of a benefit for the break condition renders a simple fatigue explanation implausible.

5. Our view, then, is that deficient inhibitory processing results in heightened $\mathrm{PI}$, which in turn impairs performance on standard span tasks. One alternative interpretation is that WM span tasks measure the total amount of resources available to an individual, and that inhibition is resource dependent (e.g., Conway \& Engle, 1994; Engle, Conway, Tuholski, \& Shisler, 1995). By this view, limitations in capacity impair inhibition, thus causing enhanced interference. Unfortunately, the present research was not aimed at addressing this chicken-egg debate.

(Manuscript received February 16, 1998; revision accepted for publication November 10, 1998.) 